\title{
Mite-Allergic Rhinitis: How to Evaluate Clinical Efficacy in Allergen-Specific Immunotherapy Trials?
}

Oliver Pfaar, $M D^{1,3, *}$ Roy Gerth van Wijk, $M D^{2}$

\author{
Address \\ ${ }^{*}, 1$ Center for Rhinology and Allergology Wiesbaden, Germany, An den Quellen 10, \\ 65189, Wiesbaden, Germany \\ Email: oliver.pfaar@allergiezentrum.org \\ ${ }^{2}$ Section of Allergology, Department of Internal Medicine, Erasmus MC, Rotter- \\ dam, The Netherlands \\ ${ }^{3}$ Department of Otorhinolaryngology, Head and Neck Surgery, Universitätsmedizin \\ Mannheim, Medical Faculty Mannheim, Heidelberg University, Mannheim, \\ Germany
}

Published online: 15 January 2015

(C) Springer International Publishing AG 2015

This article is part of the Topical Collection on Allergic Rhinitis

Keywords Allergic rhinitis • Mite allergy • Allergen-specific immunotherapy (AIT) · Clinical outcomes • Clinical trials

\section{Opinion statement}

House dust mite (HDM) allergen exposure is the most important cause of perennial allergic rhinitis and/or asthma. Although allergen-specific immunotherapy (AIT) with HDM is well established, published studies have been characterized by substantial heterogeneity in clinical endpoints. Standardization in measuring clinical efficacy is required. Moreover, when designing an AIT trial with HDM allergens, several considerations have to be taken into account. The history of HDM allergy is less clear cut than the typical history of pollen allergy. In addition, clinical features of HDM allergy may differ from those of pollen allergy. Moreover, although not easily measurable, fluctuation in allergen exposure may cause variation in symptom severity and determine the timing of assessment of clinical effects of HDM AIT.

Key points

1. A combined symptom and medication score (CSMS) is recommended as standard for the primary endpoint in future house dust mite (HDM) allergen-specific immunotherapy trials.

2. The diagnosis of HDM allergy is based on a carefully taken history in combination with sensitization to HDM allergens.

3. Eye symptoms are less prominent in patients with HDM-induced allergic rhinitis. Nasal symptoms, but not eye symptoms, should be included in the CSMS and in symptom scores as well.

4. As methods to determine allergen exposure vary and the efficacy of environmental control 
is a matter of debate, a practical approach consists of restraining patients from implementing HDM-reducing measures, such as removing carpets and introducing antimite covers, after the start of the study.

5. Efficacy evaluation in the period with the highest exposure to mites is recommended.

\section{Introduction}

One of the most relevant groups of indoor allergens in causing perennial allergic rhinitis (AR) and/or allergic asthma (AA) is found in the bodies and feces of house dust mites (HDM), with the two main species being Dermatophagoides pteronyssinus and Dermatophagoides farinae $[1,2,3 \bullet \bullet$. Besides allergen avoidance as a (secondary or tertiary) preventive treatment, which often lacks sufficient efficacy in domestic settings [4], the treatment of mite allergy is mainly based on antiallergic pharmacotherapy, as well as on allergen-specific immunotherapy (AIT) $[3 \bullet \bullet, 5 \bullet, 6]$. In a position paper on AIT, the World Health Organization (WHO) clearly highlighted the clinical efficacy of AIT in patients with HDM-induced AR and AA [7]. Within the last two decades, an increasing number of meta-analyses have demonstrated clear evidence of the clinical efficacy of this treatment for both subcutaneous and sublingual AIT (SCIT and SLIT) in HDM allergy [3••, 8-10]. However, in a recently published systematic evidence-based review on the clinical efficacy of HDM AIT, the authors emphasized "marked inter study heterogeneity in terms of

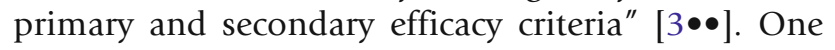

reason for this heterogeneity can be seen in specific considerations of HDM-induced allergic diseases that differentiate them from (pollen-induced) seasonal AR and AA.

Various clinical endpoints in published AIT trials for allergic rhinoconjunctivitis (ARC) were recently reviewed in a current position paper from the European Academy of Allergy and Clinical Immunology (EAACI) $[11 \bullet \bullet]$.

This article aims to (1) review examples of different outcome measures (primary and secondary), as published in the literature on HDM AIT trials (as grouped into seven domains); and (2) discuss general limitations and special considerations for clinical trials in this indication (in contrast to AIT trials in seasonal allergies). The latter include differences in the diagnosis of $\mathrm{AR}$, the clinical characteristics of patients with AR due to HDM allergy, and clinical manifestations of the disease; and limitations in measuring the allergen exposure of the patients, as well as the problem of the timing of clinical assessment of HDM AIT.

\section{Outcome measures in house dust mite allergen-specific immunotherapy trials}

\section{Symptom scores}

For AR, scoring of symptoms has been determined as clinical endpoint in several HDM AIT trials, both for the subcutaneous route (e.g., see references [12-14]) and for the sublingual route (e.g., see references [15-17]). However, there is a high grade of heterogeneity in the definition of the respective SS throughout the literature. Moreover, in some study protocols, composite-scores with nose- and lung-symptoms were used whereas a few also included conjunctival symptoms

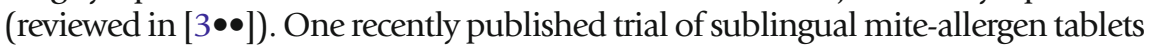
used the Average Adjusted Symptom Score (AAdSS) as a primary endpoint [18 $\bullet$.

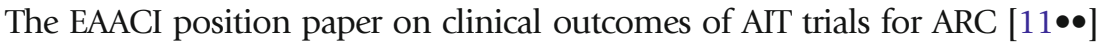
recommends a daily symptom score (dSS) in perennial AR caused by HDM, based on four nasal symptoms, as follows: itchy nose, sneezing, runny nose, and blocked 
nose, on a scale of $0-3$ points (modified from references $[19,20])$. Conjunctival symptoms are not included in this recommended scoring of the primary endpoint in AIT trials in HDM-allergic patients [11••] (see also discussion under "General limitations and special considerations for clinical trials in this indication").

(Asthma) SS have also been used as a clinical endpoint parameter in several clinical trials of HDM AIT (e.g., see references [21-24], reviewed in reference $[3 \bullet \bullet])$. As for AR, differences in asthma SS are found in comparison of these trials, with a lack of harmonized standardization of this endpoint. In the light of the need for comparability of the efficacy of HDM AIT, an international standard for the definitions of asthma SS to be used as clinical endpoints would be preferable in future trials.

Medication scores

In some clinical trials of HDM AIT in AR patients, medication scores (MS) have been used as clinical endpoints (e.g., $[13,15,18])$. As for SS, no definite standard (for both the specific class of pharmacotherapy to be administered as concomitant medication and their scoring for the MS) has been used. The EAACI position paper recommends a stepwise use of antiallergic medication and gives a standardized definition for the daily medication score (dMS) [11••], which is in line with, and is modified from, the World Allergy Organization (WAO) recommendations [19].

Some AIT trials in asthma patients have also used MS as clinical endpoints (e.g., see references [25-28]). Again, heterogeneous use of medication and scoring systems is found in the respective trial protocols, with some of them being related to and modified from a standard published by Dreborg et al. [29].

\section{Combined symptom and medication scores}

In AR, one AIT trial on HDM-allergic adult patients [30] has used a combined symptom and medication score (clinical index score [CIS]) as primary endpoint. In principle, combining symptom scores and medication scores in a composite single score as primary clinical endpoint in AIT trials is in line with the European Medicine Agency (EMA) "Guideline on the Clinical Development of Products for Specific Immunotherapy for the Treatment of Allergic Diseases" [20]. However, there is still a lack of thorough global standardization.

The EAACI position paper highlights for the primary endpoint in future AITtrials a homogeneous combined symptom and medication score (CSMS) as a simple and standardized method. This score balances both symptoms and the impact of antiallergic medication in an equally weighted ratio [11 $\bullet$ ].

In AIT trials in HDM-induced AA, composite asthma symptom and asthma medication scores have not been used as primary endpoints (reviewed in reference $[3 \bullet \bullet])$. However, in this indication, other endpoints such as controllability of asthma may be preferred as primary outcomes (see "Control of the disease").

As an important alternative to measurements of disease severity (as analyzed, for example, by SS), disease-specific "control" outcome questionnaires have recently been developed and partly validated-e.g., the Control of Allergic Rhinitis and Asthma Test (CARAT) [31•] and the Rhinitis Control Assessment Test (RCAT) [32]. However, the scores from these questionnaires have not been used as clinical endpoints in AIT trials in HDM-induced AR. 
However, in a recently published double-blind, placebo-controlled trial of SLIT in patients with HDM-induced AA, well-controlled asthma (WCA), as defined by the Global Initiative for Asthma (GINA; latest revision 2012 [33]), was used as a primary endpoint by Wang et al. [28]. Another example is a randomized, controlled trial in children with HDM-induced AA, which investigated the steroid-sparing effect of SCIT to maintain asthma control [34].

\section{Quality of life}

Quality-of-life (QoL) questionnaires were validated more than 20 years ago for both AR [35] and AA [36] in different age groups. Furthermore, a questionnaire evaluating both AR and AA in a composite format has been developed (see reference [37], reviewed in reference $[11 \bullet \bullet])$. On this basis, QoL has been evaluated in different AIT trials in HDM-induced AR [30] and AA [28].

Nasal provocation (NP; e.g., see references [38-40]) or conjunctival provocation (CP; e.g., see reference [30]) have been performed in different trials in HDM-induced AR, whereas bronchial provocation (BP) has been performed in trials in HDM-induced AA (e.g., see references $[13,25,26])$ for analyses of

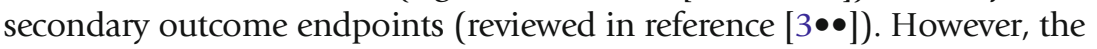
different challenge test methods have not been thoroughly standardized and validated, which should be urgently addressed in the future, not only for their clinical implications in HDM-AIT-trials (see details in reference [11••]). Environmental challenge chambers may become promising candidates for allergen provocation if thorough technical and clinical validation can be realized [41].

\section{Well days}

In the EMA "Guideline on the Clinical Development of Products for Specific Immunotherapy for The Treatment of Allergic Diseases" [20], "days with symptom control" with daily symptom scores below a "clinically justified level" are recommended as secondary endpoints for the analysis of clinical efficacy in AIT trials. As an example, one pediatric trial of SLIT in HDM-allergic children with AR used "well days" and "symptom-free days" as secondary endpoints [42]. However, it is questionable if this definition initially developed for intermittent (pollen-induced) AR can be extrapolated to persistent (HDM-induced) allergy.

\section{General limitations and special considerations for clinical trials in this indication}

\section{Diagnosis of allergic rhinitis based on house dust mite allergy}

The clinical relevance of a history matching HDM allergy is less easy to establish than the typical history of pollen allergy. Therefore, it has been suggested that a positive allergen challenge test should be part of the inclusion criteria for HDM trials $[3 \bullet \bullet]$. One problem might be that allergen challenge tests are not standardized. Moreover, the inclusion of nasal challenge tests has been used in a large trial assessing the efficacy of mattress covers [43] in children and adults with HDM allergy, but a subanalysis showed that a positive HDM challenge test did not 
substantially change the physician's diagnosis based on symptoms and sensitization to HDM [44]. Therefore, a carefully taken history in combination with sensitization to HDM allergens should be sufficient for establishing the diagnosis.

Clinical characteristics of patients with allergic rhinitis due to house dust mite allergy and the clinical manifestation of the disease

It is generally believed that rhinitis due to HDM allergy is more characterized by nasal obstruction than pollen allergy. Most AIT trials have reported the mean of different nasal symptom category scores, without showing separate nasal SS. In one small SLIT study in HDM-allergic children, low and comparable scores were reported for sneezing, nasal blockage, nasal itching and rhinorrhea [45]. In a study assessing the efficacy of montelukast and antihistamines, patients with HDM allergy reported all nasal symptom categories. Higher scores for obstruction than for sneezing and itching were reported [46]. In another SLIT study in children, rhinorrhea was seen in $89.4 \%$ and nasal obstruction in $82.3 \%$ of patients [47]. In a large study assessing QoL in patients with intermittent and persistent rhinitis, all nasal symptom categories were reported in the majority of patients with persistent rhinitis [48]. Therefore, the use of composite nasal scores is also justified in patients with HDM allergy.

Allergic conjunctivitis is often underestimated [49] and may have an important impact on QoL, even more than nasal obstruction [50]. However, in a subanalysis of children included in a retrospective HDM SLIT study, the frequency of nasal symptoms ranged from $89.4 \%$ for rhinorrhea to $61.7 \%$ for nasal pruritus, whereas the frequencies of ocular pruritus and teary eyes amounted to only $25.7 \%$ and $17.9 \%$, respectively [47]. In another HDM SLIT study, eye scores were low [42]. In line with this, many randomized, controlled AIT trials have measured only nasal symptoms without taking eye symptoms into account [12-16, 51, 39, 40,52-55], and fewer studies have included eye symptoms in the assessment of efficacy [18 $\bullet$, 42, 45, 56-59].

The recent EAACI position paper on outcome measures in AIT trials advo-

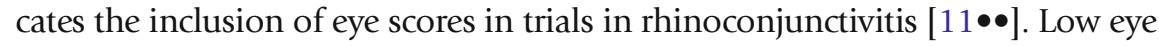
scores included in composite nasal/eye scores or combined symptom combination scores may, however, influence the effect size of treatment. For that

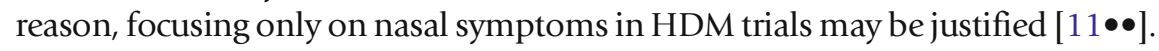
One has to bear in mind that the well-known Rhinoconjunctivitis Quality of Life Questionnaire (RQLQ) has been validated for allergic rhinoconjunctivitis [35]. Juniper et al. have also validated an instrument for perennial rhinitis [60].

Limitations in measuring allergen exposure of patients

In a few studies, measurement of HDM allergens has been performed. Exposure can be measured at baseline. In one study, relatively low levels of the major $D$. farinae allergen Der $\mathrm{f} 1$ were found in the settled dust samples. The levels ranged from non-detectable to $8 \mu \mathrm{g} / \mathrm{g}$ of dust. Mean Der $\mathrm{f} 1$ levels were similar in the active treatment and placebo groups [51]. Exposure may be monitored during the study. In two studies, no change in exposure could be observed [13, 14]. In another study, HDM exposure was expressed in guanine levels in mattresses [53]. After 24 months, the number of patients with class $2 \pm 3$ guanine levels decreased significantly in both groups $(P=0.01)$, while there was a concomitant increase in the number of patients with low guanine levels (class 1 ), indicating a marked 
change in the indoor allergen load [53]. Such a decrease in Der p 1 was also observed in a trial in asthmatics [55]. The latter study included help from an environmental consultant and the introduction of anti-mite mattresses and pillow covers before the start of the study. Although measurement and monitoring of

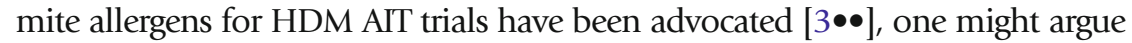
about the added value. Theoretically, a change in allergen exposure during treatment may interfere with the results of AIT. As methods to determine allergen exposure vary and the efficacy of environmental control is a matter of debate [4], a more practical approach consists of restraining patients from implementing HDM-reducing measures, such as removing carpets and introducing anti-mite covers after the start of the study.

Timing of assessment of clinical effects of allergen-specific immunotherapy for house dust mite allergy

Although peak HDM exposures are not as substantial as those seen in pollen allergy, HDM exposure might be relatively high in autumn [61]. In a Polish AIT study, differences relative to placebo were particularly evident in successive heating seasons, which corresponds to the highest period of HDM allergen exposure in Poland [56]. Also, in an Italian study, it was shown that rhinitis and medication scores were significantly lower in the AIT group than in the placebo group in October, November, and December [57]. Lower SS in the AIT group than in the placebo group in most of the winter months $(P=0.05)$ were observed in another Italian study [59]. In one study, the diary had to be filled in from November to February, when the exposure to indoor mites is expected to be greater [58]. Also, other studies have taken a specific period of inclusion or efficacy analysis into account $[18 \bullet, 40,42,52,53,55]$. Therefore, efficacy evaluation in the period with the highest exposure to mites is recommended. Moreover, assessment should always be done at yearly intervals and not at 6month intervals [16] or 18-month intervals $[14,55]$.

\section{Compliance with Ethics Guidelines}

\section{Conflict of Interest}

O. Pfaar (OP) has received research grants for his institution from ALK-Abelló (Germany/Denmark), Allergopharma (Germany), Stallergenes (Germany/France), HAL Allergy (Germany/the Netherlands), Artu Biologicals (the Netherlands), Allergy Therapeutics/Bencard (UK/Germany), Hartington (Spain), Lofarma (Italy), Novartis/Leti (Germany/Spain), GlaxoSmithKline (UK/Germany), Essex Pharma (Germany), Cytos (Switzerland), Curalogic (Denmark), Roxall (Germany), Biomay (Austria), Thermo Fisher (Germany), Circassia (UK), European Union (FP-7-Health-2013 Innovation 1), Biotech Tools s.a. (Belgium), and Meda Pharma $\mathrm{GmbH}$ (Germany); and/or he has served as an advisor and on speakers' bureaus for some of the aforementioned companies. OP has received travel grants from HAL Allergy (the Netherlands/Germany) and Allergopharma (Germany), and he is a consultant for Bencard (Germany), HAL Allergy (the Netherlands), Novartis/Leti (Germany), Meda (Germany), ALK-Abelló (Germany/Denmark) , Allergopharma (Germany), Biotech Tools s.a. (Belgium), GfK Bridgehead (UK), Navigant Consulting (USA), Sanofi (USA), Guidepoint Global Advisors (USA), Thermo Fisher (Germany) and Stallergenes (Germany/France); he is Scientific Board Member of Mobile Chamber Experts (MCX), a GA2LEN Partner. OP is the current chairman Immunotherapy 
Interest Group (IT IG) of the European Academy of Allergy and Clinical Immunology (EAACI) and is the secretary of the ENT section of Deutsche Gesellschaft für Allergologie und Klinische Immunologie (DGAKI). He has received grants for the "Spezifische Immuntherapie"-award 2014 and the "Nachwuchsförderpreis"award 2010 of the DGAKI. He is co-editor and an author of the textbook "Allergien bei Kindern und Jugendlichen" (publisher: Schattauer-Verlag, Germany) and author of different chapters of "AllergologieHandbuch" (publisher: Schattauer-Verlag, Germany) and has received payment for development of educational presentations from GlaxoSmithKline (Germany), Bencard (Germany), and Novartis (Germany). R. Gerth van Wijk (RGW) has received fees for consultancy for MSD, HAL, Novartis, and Crucell. He has received research grants from NWO, STW, Novartis, Biomay, DBV, and ALK. He has received payment for lectures from Allergopharma, payment for manuscript preparation from Chiesi, and royalties from De Tijdstroom and Bohn Stafleu van Loghum.

Human and Animal Rights and Informed Consent

This article does not contain any studies with human or animal subjects performed by any of the authors.

\section{References and Recommended Reading}

Papers of particular interest, published recently, have been highlighted as:

- Of importance

- Of major importance

1. Mosbech H. House dust mite allergy. Allergy. 1985;40(2):81-91.

2. Bousquet J, Van Cauwenberge P, Khaltaev N. Allergic rhinitis and its impact on asthma. J Allergy Clin Immunol. 2001;108(5 Suppl):S147-334.

3.• Calderon MA, Casale TB, Nelson HS, Demoly P. An evidence-based analysis of house dust mite allergen immunotherapy: a call for more rigorous clinical studies. J Allergy Clin Immunol. 2013;132(6):1322-36. Recently published comprehensive analysis of different SCIT and SLIT trials of HDM AIT in both HDM-induced AR and HDM-induced AA. The focus of this analysis was on study designs, doses, regimens, patient-reported outcomes, safety reporting, and compliance. One central finding was the high grade of heterogeneity of the endpoints used in these trials.

4. Custovic A, Wijk RG. The effectiveness of measures to change the indoor environment in the treatment of allergic rhinitis and asthma: ARIA update (in collaboration with GA(2)LEN). Allergy. 2005;60(9):1112-5.

5. $\quad$ Nelson HS. Update on house dust mite immunotherapy: are more studies needed? Curr Opin Allergy Clin Immunol. 2014;14(6):542-8.

Overview of current trials of HDM-AIT-the US/American view.

6. Calderon MA, Demoly P, Gerth van Wijk R, et al. EAACI: a European declaration on immunotherapy. Designing the future of allergen specific immunotherapy. Clin Transl Allergy. 2012;2(1):20.

7. Bousquet J, Lockey R, Malling HJ. Allergen immunotherapy: therapeutic vaccines for allergic diseases. A WHO position paper. J Allergy Clin Immunol. 1998;102(4 Pt 1):558-62.

8. Abramson MJ, Puy RM, Weiner JM. Injection allergen immunotherapy for asthma. Cochrane Database Syst Rev. 2010;8, CD001186.

9. Compalati E, Passalacqua G, Bonini M, Canonica GW. The efficacy of sublingual immunotherapy for house dust mites respiratory allergy: results of a GA2LEN meta-analysis. Allergy. 2009;64(11):1570-9.

10. Eifan AO, Calderon MA, Durham SR. Allergen immunotherapy for house dust mite: clinical efficacy and immunological mechanisms in allergic rhinitis and asthma. Expert Opin Biol Ther. 2013;13(11):1543-56.

11.• Pfaar O, Demoly P, Gerth van Wijk R, et al. Recommendations for the standardization of clinical outcomes used in allergen immunotherapy trials for allergic rhinoconjunctivitis: an EAACI position paper. Allergy. 2014;69(7):854-67.

Position paper of the EAACI on clinical endpoints in AIT trials. This position paper is structured into nine "domains" of dif-

ferent outcomes and highlights the advantages, disadvantages, and specific recommendations on each parameter. As its main achievement, the position paper recommends a homogeneous CSMS as a simple and standardized method to be used as the primary endpoint for future randomized, controlled trials of AIT for allergic rhinoconjunctivitis.

12. Varney VA, Tabbah K, Mavroleon G, Frew AJ. Usefulness of specific immunotherapy in patients with severe perennial allergic rhinitis induced by house dust mite: a double-blind, randomized, placebo-controlled trial. Clin Exp Allergy. 2003;33(8):1076-82.

13. Yukselen A, Kendirli SG, Yilmaz M, Altintas DU, Karakoc GB. Effect of one-year subcutaneous and 
sublingual immunotherapy on clinical and laboratory parameters in children with rhinitis and asthma: a randomized, placebo-controlled, double-blind, double-dummy study. Int Arch Allergy Immunol. 2012;157(3):288-98.

14. Pichler CE, Marquardsen A, Sparholt S, et al. Specific immunotherapy with Dermatophagoides pteronyssinus and D. farinae results in decreased bronchial hyperreactivity. Allergy.

1997;52(3):274-83.

15. O'Hehir RE, Gardner LM, de Leon MP, et al. House dust mite sublingual immunotherapy: the role for transforming growth factor-beta and functional regulatory T cells. Am J Respir Crit Care Med. 2009;180(10):936-47.

16. Tseng SH, Fu LS, Nong BR, Weng JD, Shyur SD. Changes in serum specific IgG4 and IgG4/IgE ratio in mite-sensitized Taiwanese children with allergic rhinitis receiving short-term sublingual-swallow immunotherapy: a multicenter, randomized, placebocontrolled trial. Asian Pac J Allergy Immunol. 2008;26(2-3):105-12.

17. Tonnel AB, Scherpereel A, Douay B, et al.: Allergic rhinitis due to house dust mites: evaluation of the efficacy of specific sublingual immunotherapy. Allergy 2004;59:491-7.

18. Bergmann KC, Demoly P, Worm M, et al. Efficacy and safety of sublingual tablets of house dust mite allergen extracts in adults with allergic rhinitis. J Allergy Clin Immunol. 2014;133(6):1608-14.

Well-performed phase II/III study to assess the efficacy and safety of two doses of HDM sublingual tablets over one treatment year and the subsequent immunotherapy-free year in 509 randomized adult patients.

19. Canonica GW, Baena-Cagnani CE, Bousquet J, et al. Recommendations for standardization of clinical trials with allergen specific immunotherapy for respiratory allergy. A statement of a World Allergy Organization (WAO) taskforce. Allergy. 2007;62(3):317-24.

20. European Medicines Agency. Committee for Medicinal Products for Human Use (CHMP). Guideline on the Clinical Development of Products for Specific Immunotherapy for The Treatment of Allergic Diseases. London: European Medicines Agency; 2008.

21. Maestrelli P, Zanolla L, Pozzan M, Fabbri LM. Regione Veneto Study Group on the "Effect of Immunotherapy in Allergic Asthma". Effect of specific immunotherapy added to pharmacologic treatment and allergen avoidance in asthmatic patients allergic to house dust mite. J Allergy Clin Immunol. 2004;113(4):643-9.

22. Olsen OT, Larsen KR, Jacobsan L, Svendsen UG. A 1year, placebo-controlled, double-blind house-dustmite immunotherapy study in asthmatic adults. Allergy. 1997;52(8):853-9.

23. Niu CK, Chen WY, Huang JL, Lue KH, Wang JY. Efficacy of sublingual immunotherapy with highdose mite extracts in asthma: a multi-center, double-blind, randomized, and placebo-controlled study in Taiwan. Respir Med. 2006;100(8):137483.

24. Ippoliti F, De Santis W, Volterrani A, et al. Immunomodulation during sublingual therapy in allergic children. Pediatr Allergy Immunol 2003;14:21621.

25. Ameal A, Vega-Chicote JM, Fernandez S, et al. Doubleblind and placebo-controlled study to assess efficacy and safety of a modified allergen extract of Dermatophagoides pteronyssinus in allergic asthma. Allergy. 2005;60(9):1178-83.

26. Garcia-Robaina JC, Sanchez I, de la Torre F, FernandezCaldas E, Casanovas M. Successful management of mite-allergic asthma with modified extracts of Dermatophagoides pteronyssinus and Dermatophagoides farinae in a double-blind, placebo-controlled study. J Allergy Clin Immunol. 2006;118(5):1026-32.

27. Pajno GB, Morabito L, Barberio G, Parmiani S. Clinical and immunologic effects of long-term sublingual immunotherapy in asthmatic children sensitized to mites: a double-blind, placebo-controlled study. Allergy. 2000;55(9):842-9.

28. Wang L, Yin J, Fadel R, Montagut A, de Beaumont $\mathrm{O}$, Devillier P. House dust mite sublingual immunotherapy is safe and appears to be effective in moderate, persistent asthma. Allergy. 2014;69(9):1181-8.

29. Dreborg S, Agrell B, Foucard T, Kjellman NI, Koivikko A, Nilsson S. A double-blind, multicenter immunotherapy trial in children, using a purified and standardized Cladosporium herbarum preparation. I Clinical results Allergy. 1986;41(2):131-40.

30. Riechelmann H, Schmutzhard J, van der Werf JF, Distler A, Kleinjans HA. Efficacy and safety of a glutaraldehydemodified house dust mite extract in allergic rhinitis. Am J Rhinol Allergy. 2010;24(5):e104-9.

31. Fonseca JA, Nogueira-Silva L, Morais-Almeida M, et al. Control of Allergic Rhinitis and Asthma Test (CARAT) can be used to assess individual patients over time. Clin Transl Allergy. 2012;2(1):16.

Report on recently developed disease-specific AR "control" questionnaires, which may become useful candidates for (clinical) endpoints in future AIT trials.

32. Nathan RA, Dalal AA, Stanford RH, et al. Qualitative development of the Rhinitis Control Assessment Test (RCAT), an instrument for evaluating rhinitis symptom control. Patient. 2010;3(2):91-9.

33. Global Initiative for Asthma (GINA). Global strategy for asthma management and prevention. December 2012 update of the NHLBI/WHO Workshop Report 1995. Available from: http://www.ginasthma.org/. 2012.

34. Zielen S, Kardos P, Madonini E. Steroid-sparing effects with allergen-specific immunotherapy in children with asthma: a randomized controlled trial. J Allergy Clin Immunol. 2010;126(5):942-9.

35. Juniper EF, Guyatt GH. Development and testing of a new measure of health status for clinical trials in rhinoconjunctivitis. Clin Exp Allergy. 1991;21(1):7783. 
36. Juniper EF, Guyatt GH, Epstein RS, Ferrie PJ, Jaeschke R, Hiller TK. Evaluation of impairment of health related quality of life in asthma: development of a questionnaire for use in clinical trials. Thorax. 1992;47(2):76-83.

37. Baiardini I, Pasquali M, Giardini A, et al. Rhinasthma: a new specific QoL questionnaire for patients with rhinitis and asthma. Allergy. 2003;58(4):289-94.

38. Dokic D, Schnitker J, Narkus A, Cromwell O, Frank E. Clinical effects of specific immunotherapy: a two-year double-blind, placebo-controlled study with a one year follow-up. Prilozi. 2005;26(2):113-29.

39. Gabriel MS, Ng HK, Allan WG, Hill LE, Nunn AJ. Study of prolonged hyposensitization with D. pteronyssinus extract in allergic rhinitis. Clin Allergy. 1977;7(4):325-39.

40. Blainey AD, Philips MJ, Ollier RJ, Davies RJ. Hyposensitization with a tyrosine adsorbed extract of Dermatophagoides pteronyssinus in adults with perennial rhinitis. Allergy. 1984;39:521-8.

41. Rösner-Friese K, Kaul S, Vieths S, Pfaar O. Environmental exposure chambers in allergen immunotherapy trials: current status and clinical validation needs (in press). J Allergy Clin Immunol 2014; doi: 10.1016/ j.jaci.2014.10.045.

42. de Bot CM, Moed H, Berger MY, et al. Sublingual immunotherapy not effective in house dust miteallergic children in primary care. Pediatr Allergy Immunol. 2012;23(2):150-8.

43. Terreehorst I, Hak E, Oosting AJ, et al. Evaluation of impermeable covers for bedding in patients with allergic rhinitis. N Engl J Med. 2003;349(3):237-46.

44. Terreehorst I, Oosting AJ, Tempels-Pavlica Z, et al. Prevalence and severity of allergic rhinitis in house dust mite-allergic patients with bronchial asthma or atopic dermatitis. Clin Exp Allergy. 2002;32(8):1160-5.

45. Aydogan M, Eifan AO, Keles S, et al. Sublingual immunotherapy in children with allergic

rhinoconjunctivitis mono-sensitized to house-dustmites: a double-blind-placebo-controlled randomised trial. Respir Med. 2013;107(9):1322-9.

46. Ciebiada M, Gorska-Ciebiada M, DuBuske LM, Gorski P. Montelukast with desloratadine or levocetirizine for the treatment of persistent allergic rhinitis. Ann Allergy Asthma Immunol. 2006;97(5):664-71.

47. Trebuchon F, Lheritier-Barrand M, David M, Demoly P. Characteristics and management of sublingual allergen immunotherapy in children with allergic rhinitis and asthma induced by house dust mite allergens. Clin Transl Allergy. 2014;4:15.

48. Bousquet J, Neukirch F, Bousquet PJ, et al. Severity and impairment of allergic rhinitis in patients consulting in primary care. J Allergy Clin Immunol. 2006;117(1):158-62.

49. Klossek JM, Annesi-Maesano I, Pribil C, Didier A. The burden associated with ocular symptoms in allergic rhinitis. Int Arch Allergy Immunol. 2012;158(4):411-7.
50. Bousquet PJ, Demoly P, Devillier P, Mesbah K, Bousquet J. Impact of allergic rhinitis symptoms on quality of life in primary care. Int Arch Allergy Immunol. 2013;160(4):393-400.

51. Bush RK, Swenson C, Fahlberg B, et al. House dust mite sublingual immunotherapy: results of a US trial. J Allergy Clin Immunol. 2011;127(4):974-81. e1-7.

52. Bahceciler NN, Isik U, Barlan IB, Basaran MM. Efficacy of sublingual immunotherapy in children with asthma and rhinitis: a double-blind, placebo-controlled study. Pediatr Pulmonol. 2001;32(1):49-55.

53. Guez S, Vatrinet C, Fadel R, Andre C. House-dust-mite sublingual-swallow immunotherapy (SLIT) in perennial rhinitis: a double-blind, placebo-controlled study. Allergy. 2000;55(4):369-75.

54. Mungan D, Misirligil Z, Gurbuz L. Comparison of the efficacy of subcutaneous and sublingual immunotherapy in mite-sensitive patients with rhinitis and asthma-a placebo controlled study. Ann Allergy Asthma Immunol. 1999;82(5):485-90.

55. Pham-Thi N, Scheinmann P, Fadel R, Combebias A, Andre C. Assessment of sublingual immunotherapy efficacy in children with house dust mite-induced allergic asthma optimally controlled by pharmacologic treatment and mite-avoidance measures. Pediatr Allergy Immunol. 2007;18(1):47-57.

56. Bozek A, Ignasiak B, Filipowska B, Jarzab J. House dust mite sublingual immunotherapy: a doubleblind, placebo-controlled study in elderly patients with allergic rhinitis. Clin Exp Allergy. 2013;43(2):242-8.

57. Marcucci F, Sensi L, Di Cara G, et al. Three-year followup of clinical and inflammation parameters in children monosensitized to mites undergoing sub-lingual immunotherapy. Pediatr Allergy Immunol.

2005;16(6):519-26.

58. Passalacqua G, Pasquali M, Ariano R, et al. Randomized double-blind controlled study with sublingual carbamylated allergoid immunotherapy in mild rhinitis due to mites. Allergy. 2006;61(7):849-54.

59. Passalacqua G, Albano M, Fregonese L, et al. Randomised controlled trial of local allergoid immunotherapy on allergic inflammation in miteinduced rhinoconjunctivitis. Lancet. 1998;351(9103):629-32.

60. Juniper EF, Guyatt GH, Andersson B, Ferrie PJ. Comparison of powder and aerosolized budesonide in perennial rhinitis: validation of rhinitis quality of life questionnaire. Ann Allergy. 1993;70(3):225-30.

61. van der Heide S, de Monchy JG, de Vries K, Bruggink TM, Kauffman HF. Seasonal variation in airway hyperresponsiveness and natural exposure to house dust mite allergens in patients with asthma. J Allergy Clin Immunol. 1994;93(2):470-5. 